\title{
Arbor
}

\section{Lenguaje y conocimiento científico: perspectivas para una educación española}

\section{Francisco González de Posada}

Arbor CLXXIII, 681 (Septiembre 2002), 105-127 pp.

Con el horizonte de una marcada preocupación ante el problema que se le plantea a la Lengua española por el abandono a que la somete el imperio de la Lengua inglesa en la creación y uso de términos científicos, y por el exilio lingüístico de los científicos españoles, en este trabajo se tratan los siguientes problemas: 1) la dificultad del Lenguaje para la Filosofía, para la conceptualización y la designación; 2) la participación de los científicos españoles en las tareas de la Academia Española, particularizada en Leonardo Torres Quevedo y Blas Cabrera; 3) el panorama del Español científico ante la creación y el uso de términos; para terminar con 4) la exposición de algunas vías para detener el proceso de deterioro de la Lengua española.

El título que se me ha asignado consiste intrínsecamente en una expresión copulativa: «lenguaje» $\mathrm{y}$ «conocimiento científico». Contextualmente se inserta en un monográfico dedicado a «educación» referida a España, en español y para españoles, es decir, «española». En consecuencia, los matices y la orientación de la expresión copulativa titular deben concretarse en la lengua española y dirigirse a la educación. En esta tarea nos situaremos. Con un aviso: considero ciencia a las ciencias de verdad: matemática, física, química, biología y afines. $\mathrm{Y}$ un lamento: ¿por qué tanta obsesión en otros saberes -tan dignos como los científicos- por ser 
considerados ciencia? Desde mi condición intelectual híbrida que cree separar naturalmente ámbitos que considera como obviamente separables, este problema no existe.

No obstante, el marco delimitado, como puede comprenderse fácilmente, es de una extensión inabarcable y serían muchísimos los temas a desarrollar y los problemas a enunciar. Pero me referiré, y sin penetrar a fondo en ninguno de ellos, a los siguientes: 1) acerca del Lenguaje en sí: en torno a Wittgenstein, en la celebración del Cincuentenario de su muerte; 2) sobre las relaciones entre los científicos españoles y la lengua española: en torno a Blas Cabrera, haciendo un poco de historia de la primera mitad del siglo XX; 3) el Español científico: .cerca de la creación y el uso de términos; y finalmente 4) unas sugerencias de acciones.

\section{En el Cincuentenario de Wittgenstein: el filosofar sobre el Lenguaje}

En el año 2001 recientemente ido se ha celebrado el Cincuentenario de la muerte de Ludwig Wittgenstein (Viena, 1889; Cambridge, 1951). El filósofo austriaco es cita obligada cuando se enfrenta uno con temas de la naturaleza del que se nos ha propuesto. Wittgenstein es uno de los filósofos más significativos del siglo pasado, y dejó su principal impronta en el ámbito que puede denominarse del análisis lingüístico, de la lógica del lenguaje. Parece, pues, oportuno que le dediquemos el primer capítulo de este breve estudio por razones intrínsecas y también de actualidad conmemorativa ${ }^{1}$.

Wittgenstein pasa también por ser, para algunos -a mi juicio, demasiados-, el cénit del filosofar si no de la Filosofía. Aunque no pertenezco a este grupo, reconozco que la obra del tan afamado como desgraciado filósofo merece una atención especial en su condición de filósofo del lenguaje, ámbito en el que se ha situado en la cima.

\subsection{En torno a la Filosofía}

Wittgenstein fue autor de dos obras singulares de la filosofía producida en el siglo XX: el Tractatus Logico-Philosophicus (1921) y las Investigaciones filosóficas (1953), que se consideran que revolucionaron los cimientos de la Filosofía en la plenitud del siglo XX.

Como referente de estas líneas se utiliza un trabajo tan sorprendente como demoledor. El País conmemoró el cincuentenario de la muerte en el 
«extraordinario» Babelia del 28 de abril de 2001, con unos artículos de Isidoro Reguera. El texto principal puede caracterizarse como 'de los tres despueses'. Reproduzcamos tres afirmaciones rotundas de sentidos negativos.

Primera: «Después de él [de Wittgenstein] ya no se puede pensar como antes. Conmovió los fundamentos y certezas tradicionales del pensar por excelencia, la filosofía, y a su destrucción dedicó consciente y heroicamente la vida».

Segunda: «Después de Wittgenstein no se puede hacer filosofía como antes, o no se puede hacer filosofía de ningún modo, si se la entiende como antes».

Y tercera: «Desde Wittgenstein nada puede ser universal, no puede haber principios categoriales o imperativos generales, hay que despedirse definitivamente de todo fundamento».

Con cierto atrevimiento pueden hacerse dos consideraciones: una de carácter consecuencial de las precedentes afirmaciones de los despueses, y otra de manifiesta discrepancia.

En primer lugar, la consecuencial, que puede catalogarse, a los efectos que se desean destacar, como relativa al ambiente sociológico de la Filosofía en España. ¡Así nos ha ido! ¡Así nos va! Tanto tanto que no es que nos dejara sin Filosofía, con mayúsculas, sino que convenció a (muchos de) los filósofos profesionales de que no podía existir Filosofía; pero como había filósofos profesionales que tenían que trabajar contribuyó de manera singular, aunque obviamente sin proponérselo, al pulular de las «filosofías-de» y de las «filosofías adjetivadas» (formalmente, no personalmente), todas con minúsculas, y de casi todo, como si el hecho concreto tempóreo de que no pudiera existir una sola y auténtica Filosofía obligara a que tuvieran que existir muchas, cuantas más mejor. Así los planes de estudio de la mayoría de nuestras Facultades de Filosofía manifiestan que se estudian en ellas casi todos estos tipos de filosofía de casi todo menos propiamente Ciencia (al modo filosófico propuesto por Ortega en su Misión de la Universidad) y Filosofía, menos Física (a dicho modo filosófico) y Metafísica.

En segundo lugar, la discrepancia. Puede afirmarse rigurosamente que se ha hecho Filosofía, con mayúsculas, después de Wittgenstein y no de menor rango que la que se hizo antes de él. Ahí están mis maestros, que también lo son de otros muchos, Xavier Zubiri y Mario Bunge. Pero, obviamente, nuestra convicción no quita mérito alguno al filósofo austríaco ni el merecido reconocimiento al trabajo del autor del artículo conmemorativo que me honro -honrándolo a él- en utilizar como referente. Puede avanzarse un poco más para que se entienda un poco mejor 
otro aspecto de lo que deseo dejar constancia expresa y múltiple. Reguera cita junto a Wittgenstein a otros tres pensadores germánicos de indudable relevancia en la Historia de la Filosofía, ya reconocidos e integrados en el plano académico, como son Freud, Nietzsche y Heidegger, y marginalmente a Russell. Sin duda me perdonará que haya citado ya a dos pensadores hispánicos, más recientes, aún no integrados en el saber profesionalizado del ámbito académico y para cuyas citas, y además con el énfasis que acostumbro, hace falta más osadía y quizás mucho atrevimiento; con los suyos no se naufraga, ciertamente, pero conviene 'ir sabiendo' que con los míos tampoco. No debemos avergonzarnos de leer en español, ni tener miedo de citar a los que son españoles o escriben en español. Y esto tiene mucha importancia intrínseca pero también y especialmente en y para el trabajo que se me ha solicitado.

En síntesis, puede afirmarse que hoy sí hay -y puede haber, y debe haber, y es conveniente que haya- Filosofía, con mayúsculas, Filosofía primera o Metafísica. Otro problema es que no la hubiera en tiempos de Wittgenstein y si se me apura que ni siquiera pudiera haberla. Pero Filosofía primera o Metafísica no tiene por qué ser una doctrina, conviene que sea sólo respuesta al (a los) problema (s) fundamental (es). Por mi parte, me atrevo a hablar, como es mi obligación, en el ámbito de la $\mathrm{Na-}$ turaleza y de las Ciencias de la Naturaleza y puedo decir que es posible -y no sólo deseable- a la luz de las concepciones actuales de las ciencias físicas y biológicas una Metafísica de la Naturaleza, y aún más, que existe: la conjunción de Sobre la esencia y Estructura dinámica de la Realidad de Zubiri que, como digo, no es una doctrina sino lo que clásicamente podría considerarse como una Filosofía de la Naturaleza. (Denominándola con más rigor -ya que no se corresponde propiamente a ella, dado que abarca bastante más- sería Metafísica de la Realidad).

Wittgenstein sí constató la realidad intelectual de su época: el vacío filosófico, por una parte, y la imposibilidad (la suya y la de sus coetáneos) de llenarlo, por otra. Las ciencias físicas y biológicas no permitieron más en aquellos momentos de crisis generalizada del pensamiento.

Pero en la década de los cincuenta se cerraron las radicales revoluciones de la Física al mismo tiempo que se abrían las también radicales de la biología. Con Wittgenstein no se acabó el filosofar. Y contra sus criterios no sólo puede hacerse Filosofía sino que se ha hecho. No será la última vez que deba hacerse dadas las ignorancias científicas actuales y las revoluciones en marcha, y es de esperar, en consecuencia, que también en el futuro se haga.

No es extraño que Zubiri no le prestara atención al filósofo austríaco. Y sí se la dedicara a la Matemática, a la Física y a la Biología junto a las 
Lenguas. Eligió otro camino, sin duda más duro y difícil pero abierto hacia una meta que se ve aunque nunca se alcanzara, alcanzándola.

\subsection{En torno al Lenguaje}

Pero, la filosofía -si así se nos deja llamarla- de Wittgenstein pasa por ser, con razón, entre otras cosas, praxis analítica y crítica del lenguaje; es decir, se presenta por ello como de sumo interés para el tema que nos reúne.

La filosofía es para Wittgenstein, según Reguera: 1) praxis analítica; 2) crítica del lenguaje; 3) estilo de vida y de pensar; y 4) no es una doctrina. Esto más que filosofía parece que corresponde al filosofar, a la acción intelectual filosófica, al ejercicio de la racionalidad.

Y Wittgenstein detectó, mejor que nadie hasta entonces, la problemática inherente al lenguaje, su irracionalidad, su inducción a la confusión. En este sentido, su obra adquiere un extraordinario y significativo valor, pero sólo en este ámbito.

$\mathrm{Si}$ en el parágrafo anterior se ha manifestado una radical discrepancia, ahora no hay inconvenientes para tomar prestado del dutor del trabajo conmemorativo de El País unas frases haciéndolas en algún sentido nuestras, como introducción para la posterior exhibición de un elenco de problemas.

«Toda la racionalidad que puede haber pertenece al lenguaje, y el lenguaje consiste en mil juegos y contextos diferentes, con reglas diferentes cada uno. El uso diario de las palabras genera todo y cualquier sentido en el mundo. Cualquier significado y sentido de las cosas es relativo siempre a esta modesta e infranqueable coyuntura. Lo demás son fantasmas. La duda creadora de problemas filosóficos trascendentales desaparece en cuanto uno retorna al punto de vista del sentido común, y tales problemas con ella».

Demasiado fuerte por lo que respecta a la racionalidad, a los problemas filosóficos pero no precisamos más en estos ámbitos.

$\mathrm{Y}$ por lo que respecta a la crítica del lenguaje estamos de acuerdo, ¡cómo no!, con Wittgenstein y con Reguera, aunque fueran precisos algunos matices.

Dos consideraciones complementarias se presentan como de sumo interés.

Primera, como glosa y recuerdo de las palabras de Wittgenstein: "Desconfianza de la gramática es la primera condición para filosofar».

Segunda, para el laudator: «antes de saber si es verdadero o falso lo que decimos hay que saber si siquiera decimos algo cuando hablamos. Y 


\section{Francisco González de Posada}

si decimos algo, qué decimos y desde dónde lo hacemos, desde qué juego lingüístico, qué contexto, qué forma de vida».

Podemos dar por concluida esta densa, quizás extensa y aparentemente periférica presentación del problema fundamental que nos atañe. Es momento de centrar nuestra atención en el cuadro sintético adjunto en el que se distinguen al modo clásico ${ }^{2}$, en primer lugar, los niveles correspondientes a la Realidad y al ámbito de la Lógica, subdividido éste a su vez en dos subniveles: el conceptual y el lingüístico (mediante 'términos' $\mathrm{y}$, en su caso, 'expresiones' para designar a referentes y/o conceptos) ${ }^{3}$.

\begin{tabular}{|c|c|c|}
\hline \multicolumn{2}{|c|}{ REALIDAD } & REFERENTE \\
\hline \multirow{3}{*}{ LÓGICA } & Conceptual & Concepto \\
\cline { 2 - 3 } & Lingüístico & Término \\
\hline
\end{tabular}

De esta manera clásica, y a falta de consideraciones críticas y de detalles, puede decirse que el concepto describe el referente (la cosa, el objeto) y el término lo designa constituyendo referente, concepto y término la trilogía de elementos fundamentales que hemos de considerar. Y con los tres tiene que habérselas «nuestro» humano lénguaje.

Pues bien, aunque sea sólo como síntesis puede hacerse un breve catálogo inicial de problemas concretos, ahora con la única finalidad de clasificarlos y denominarlos.

Primero, la importancia del lenguaje.

Segundo, la dificultad del lenguaje, de su uso adecuado, por su esclavitud al contexto, complejo, versátil, tempóreo, espacial.

Tercero, la necesidad del lenguaje, que se hace inexcusable.

Y cuarto, el desplazamiento continuo de nuestra atención desde el término al concepto y viceversa, el tránsito del nivel lógico lingüístico al nivel lógico conceptual (que, en parte principal, es también lingüístico).

Antes de acabar esta primera parte, y aunque sólo sea a título de pequeño apéndice, dado que este tema inicial afecta al lenguaje con tratamiento filosófico, deseo dejar otras dos pinceladas como esbozo. Una relativa a Ortega, usador como pocos del español, maestro del arte de escribir y filósofo. Otra, para citar de nuevo a Zubiri, ahora considerándolo como creador, quizás el que más, de lenguaje español. Numerosas voces adquieren con él nuevas acepciones, $y$, sin rubor, genera una pléyade de nuevos términos con los que designa nuevos conceptos referidos 
a nuevas visiones de la realidad y consecuencias de su estudio de la misma.

Finalmente, y como resumen, debe afirmarse que el Lenguaje es un problema para la Filosofía, pero no problema de la Filosofía que hartos problemas tiene o, mejor dicho, que sólo tiene problemas y que ella misma ya es problema y que como problemas enuncia y estudia sus quehaceres. Pero el Lenguaje no sólo es problema para la Filosofía sino que lo es también intrínsecamente -aunque de manera menos profunda- para la Ciencia.

Pero aquí y ahora la perspectiva que interesa es otra: la de los problemas que la Ciencia y la Técnica en sus vertiginosos desarrollos crean extrínsecamente a las lenguas, y, en concreto, a la Lengua española.

\section{Los científicos españoles y la Lengua: en torno a Blas Cabrera}

Está bien lo anterior, pero debe escribirse no sólo sobre el Lenguaje (vocabulario y gramática), su problemática y su inexcusibilidad, sino sobre el Lenguaje científico y en español. Se tratarán en adelante algunas cuestiones puntuales. Cuestiones de historia, pero de historia reciente, de aproximación a la actualidad en la relación entre científicos españoles y la lengua. Centraremos la atención en la figura de Blas Cabrera por mediación de su Discurso de ingreso en la Academia Española.

\subsection{Científicos académicos de la Española}

Hagamos, en primer lugar, un excurso para recordar a algunos de los más notables científicos españoles del siglo XX que tuvieron unas relaciones especiales con la lengua, refiriéndonos sólo a los más famosos que ingresaron en la Real Academia Española: serán los más conocidos y quienes adquirieron mayor responsabilidad. En consecuencia, no se pretende hacer un catálogo de los científicos españoles que se han ocupado de este tema, tampoco hacer un pequeño tratado de historia. Y menos aún hacer crítica del presente respecto de la atención que aquellos dedicaron y de la que dedican por exceso o defecto los actuales, y la que a ellos le dedican los restantes. Como contexto del trabajo lingüístico de aquéllos, conviene recordar que, en general, los científicos españoles mayoritariamente dedican poca atención a la lengua española, ya que parece que está en paralelo la importancia que se autoconceden con el desprecio que al menos implícitamente hacen de nuestra lengua. 
El catálogo integraría a José de Echegaray, José Rodríguez Carracido, Leonardo Torres Quevedo, Santiago Ramón y Cajal, Blas Cabrera, Julio Rey Pastor, Esteban Terradas, Julio Palacios, Gregorio Marañón y Pedro Laín Entralgo. La opción personal elegida se ha orientado principalmente por tres personajes singulares de la ciencia española: Leonardo Torres Quevedo, Blas Cabrera y Julio Palacios.

En esta ocasión, 2002, debe tenerse un nuevo recuerdo de Torres Quevedo con motivo del centenario de su participación en «La conquista del aire» (González de Posada y González Redondo 2002) y glosar de manera específica las relaciones de Blas Cabrera con la Academia Española, utilizando estas relaciones como fuente principal para hacer un nuevo y breve catálogo de problemas ya más próximo al interés prescrite.

Torres Quevedo puede considerarse como el pionero del actual e importante Vocabulario Científico y Técnico de la Real Academia de Ciencias Exactas, Físicas y Naturales. En ocasión anterior he escrito sobre Torres Quevedo y la Lengua. En Leonardo Torres Quevedo (1992), le dediqué el capítulo final destacando cuatro aspectos de la obra del genial inventor español. Primero, como creador de una nueva lengua simbólica para la descripción de las máquinas con la propuesta de algunas reglas para su gramática y algunos símbolos de su diccionario. Segundo, como usuario ejemplar de la lengua ordinaria (española y francesa) en ciencia y técnica. Tercero, como ideador -y proponente de la realización- de un Diccionario Tecnológico Español en el marco de una «Unión Internacional Hispano Americana de Bibliografía y Tecnología Científicas». Y cuarto, como académico de la Lengua. A esta obra se remite al lector para más detalles.

\subsection{Notas en torno a Cabrera}

\section{1) Su participación en el Diccionario}

Blas Cabrera, que fue elegido académico de la Española para suceder a Cajal el 14 de diciembre de 1934, tomó cierto interés en las tareas de la Academia, aunque no consta que fuera mucho. Incluso antes de tomar posesión de su sillón, escribió una carta el 18 de febrero de 1935 a Emilio Cotarelo, secretario, colaborando en la edición del Diccionario, entonces en elaboración:

«Si no encuentran Uds. que sea excesivamente técnica pudiera adoptarse la siguiente definición de la palabra Protón:

"Núcleo del átomo de hidrógeno donde se concentra la casi totalidad de su masa material y que tiene una carga eléctrica positiva 


\section{Lenguaje y conocimiento científico}

numéricamente igual a la negativa del electrón. Debe su nombre a que se le considera el elemento generador de todos los demás átomos".

Se me ocurre si no consideran Udes. conveniente incluir en el diccionario los nombres: Neutrón, Positrón, Deutón de importancia también grande.

Está siempre a la disposición de la Academia».

Pueden apreciarse tres aspectos:

a) La dificultad de toda definición de un término nuevo (el protón ya era antiguo), intrínseca y por razones de lugar y tiempo, que Cabrera salva bastante bien.

b) El retraso de los términos científicos para penetrar en los diccicnarios de la Lengua.

c) El interés de Cabrera por acelerar este proceso de presencia oficial sugiriendo introducir otros términos relativos, en este caso, al neutrón y positrón que se habían 'descubierto' sólo unos dos años antes.

\section{2) Título y tema de su Discurso}

Cabrera leyó el preceptivo Discurso de ingreso el 26 de enero de 1936, de título harto significativo para nuestro tema: «Evolución de los conceptos físicos y lenguaje».

Acerca de la Realidad, que en el ámbito de la Física puede sustituirse por Naturaleza, suelo decir que, de acuerdo con Newton, la realidad «es lo que es», «es como es» («y tanto mejor si puede expresarse matemáticamente», que no siempre se puede); y, más allá -es decir, más acá- de Newton, que la realidad «funciona como funciona», y, en general, lo que sería más correcto, pero aún faltan términos precisos lingüísticos, «esfunciona o funciona-es como es-funciona o funciona-es» (González de Posada 2001). Y esto, que sólo es un ejemplo, entre muchos posibles, pero que no debe olvidarse, constituye un gran problema, que presenta dos caras: la deficiencia y la insuficiencia del lenguaje actual para expresar el conocimiento científico.

De manera análoga, y también como otro caso tan elemental como radical para describir la situación presente, puede afirmarse que no existen términos que, por ejemplo en forma interrogativa, sustituyeran al ¿dónde-cuándo? o ¿cuándo-dónde? que exigiría la Relatividad Especial contextualizada formalmente en el espacio-tiempo de Minkowski en el que se sitúan los sucesos que considera esta relatividad ${ }^{4}$. Y podría llenarse todo un diccionario que mostrara las insuficiencias de términos 
para designar nuevos conceptos. Con esa insuficiencia se presentaría el problema complementario ya citado, la deficiencia de los mismos como objeto de definiciones; no sólo número (sería la insuficiencia) sino también la cualidad.

El cuadro esquemático del parágrafo anterior lo conocía perfectamente Cabrera aunque no lo utilizara formalmente. Y a él se refiere en su Discurso. Los conceptos físicos evolucionan, cambian, se modifican, adquieren nuevos contenidos, presentan diferentes y más o menos notas caracterizadoras. Los conceptos científicos se refieren a la Naturaleza y se denominan mediante voces o términos lingüísticos.

No obstante el Discurso no es propiamente tan técnico desde un punto de vista formal filosófico sino que propiamente es más bien de naturaleza histórica. Tratamiento de la evolución de la Física desde Newton hasta sus días con asombrosa actualidad: la evolución de las concepciones físicas acerca de la Naturaleza, principalmente en los dos planos tradicionales de la Filosofía de la Naturaleza y de la Física: Cosmología y naturaleza de la materia.

Por lo que respecta a la Cosmología -el estudio, imagen y concepción del Universo- describe la evolución que corresponde al tránsito de las teorías newtonianas de la Dinámica y de la Gravitación hasta la Relatividad General de Einstein y los modelos de expansión del Universo, dedicando una especial atención a las concepciones del espacio y el tiempo newtonianos asociados a las categorías de la conciencia humana y al espacio-tiempo de Einstein con unas singulares consideraciones acerca de la ins.; cia mental.

\section{3) Ideas de Cabrera sobre la Academia}

En la «Presentación» de su Discurso escribe que la Academia «procura el perfeccionamiento de la lengua española, enriqueciéndola como instrumento de expresión de las ideas». Es decir: a) perfeccionamiento; b) enriquecimiento. Mejorar la expresión de las ideas e incrementar en la medida que sea necesaria el catálogo de términos. En el lenguaje popular y a los efectos de la vida tradicional un diccionario estático puede tener sentido. Las continuas revoluciones científicas y técnicas obligan a una atención y a una decisión valiente para abarcar con términos adecuados las nuevas realidades descubiertas y los nuevos conceptos elaborados. Ardua y extensa tarea la que corresponde a la Academia y, sobre todo, la que corresponde a los académicos científicos. 


\section{Lenguaje y conocimiento científico}

4) La Física y la lengua española: catálogo de problemas españoles

Dadas la riqueza de ideas y la claridad de su exposición podemos limitarnos a enumerar y enunciar un conjunto de problemas que exhibe Cabrera en su Discurso destacando partes de sus párrafos o bien explicitando ideas concretas, sin comentarios complementarios que considero innecesarios en este punto.

1) La falta radical de tradición española en las Ciencias físicas que tuvo como consecuencia «la falta de colaboración española en el desarrollo de la Física, con la consiguiente depresión de la cultura».

2) El abandono y descuido del español: «[...] allá en los últimos años del siglo XIX y los albores del XX [...] los amantes de las ciencias físicas nos vimos forzados a descuidar la lengua materna en términos tales que casi llegamos a desconocerla. La simple información del estado de estos conocimientos era necesaria adquirirla en lengua extranjera, pues ni siquiera disponíamos de las buenas traducciones españolas de obras fundamentales que hoy abundan [...].

¡Y si al menos se nos hubiese colocado en condiciones de utilizar idiomas extranjeros!».

3) Las ideas nuevas están en otras lenguas. En tiempos del joven Cabrera en francés y alemán; en el de su Discurso se abre camino el inglés como lengua principal hacia la práctica unicidad de la actualidad.

4) Ni siquiera existían buenas traducciones, a principios de siglo, que según él sí las había en su madurez, 1936.

5) La dificultad de expresar la ciencia en español que tenían los científicos españoles: «La versión en lengua materna de las ideas ya captadas planteaba problemas casi insolubles para quienes a la par carecíamos de conceptos suficientemente claros y de dominio del espíritu del idioma a que habíamos vuelto la espalda».

6) La contribución de las ciencias al desarrollo de las lenguas: «Es la lengua producto de la cultura toda de los pueblos que la hablaron, pues en ella va quedando el sedimento de la vida intelectual de las generaciones pasadas. Los diversos órdenes de ideas influyen en la medida de su intervención en la vida ordinaria. Es, por tanto, lógico que la literatura y las artes en general ocupen el primer rango por la importancia principalísima que en estos aspectos de la actividad intelectual tiene la vida interna del espíritu humano, pero sería un error fundamental desconocer la contribución de las ciencias en la formación del arsenal de medios de expresión, para los sentimientos e ideas elaborados por la inteligencia. Téngase presente que en cuanto el hombre se sintió espectador de la naturaleza aspiró a interpretarla y comprenderla, utilizando las ventajas 
que de este conocimiento pudo deducir para mejorar su condición vital. De este modo nacen los diversos capítulos de la ciencia cuyas aportaciones al patrimonio cultural contenido en el lenguaje son proporcionadas a la participación en que sus problemas afectan a los intereses y gustos de la humanidad, pues el lenguaje se constituye a impulsos de la necesidad de comunicar las ideas que reflejan las preocupaciones individuales».

7) La necesidad de introducir nuevos términos: su evolución y adecuación. «En primer término, las diversas técnicas que el hombre ha ido creando para auxiliarse en la satisfacción de sus necesidades físicas exigen la introducción de palabras para designar los nuevos artificios, cualidades o modos de actuar. La celeridad de su evolución durante el último siglo ha creado un problema de cierta trascendencia que ha obligado a atender con esmero a la selección de los términos adecuados. $\mathrm{Y}$ así en vez de dejar libre al genio del lenguaje se crean comisiones internacionales de especialistas que determina el vocabulario. Cr mo es lógico en ellas tienen la hegemonía los pueblos que han contribuido más intensamente al progreso técnico».

8) El problema de los barbarismos. «Quienes se limitan a utilizar sus ventajas suelen cargar el idioma nacional de barbarismos innecesarios y excesivos sin ninguna atención al espíritu de aquél, que en ocasiones podría respetarse con sólo seleccionar convenientemente el origen del vocablo aceptado, y aun a veces se olvidan palabras bien castizas cuya significación cuadra exactamente con la idea que se quiere representar. En el mundo científico y en el industrial, sin distinción de razas, es frecuente aceptar un término extranjero para la designación de un fenómeno o de una de sus cualidades características, prescindiendo de palabras del propio idioma que designan exactamente la misma idea. Es ello un tributo pagado a los autores del descubrimiento y por consecuencia un poco excepcional».

«El caso que criticamos es la invasión en masa de vocabulario de una técnica que es consecuencia del usufructo de sus ventajas materiales sin contribuir a su progreso. [...] En la industria como en las demás actividades humanas lo esencial son las ideas, cuya realización se encomienda a los organismos sociales adecuados para darles efectividad».

9) El alto grado de exigencia idiomática de las ciencias puras: «Las exigencias idiomáticas de la ciencia pura son más importantes que las procedentes de a vida industrial, porque se refiere a concepciones muy fundamentales que requieren un modo de expresión adecuado para transmitirlas fielmente. Concretando más puede afirmarse que las Ciencias físicas ocupan el primer rango como promotores de cultura moderna». 
10) El daño a la Lengua española. «Así se comprende el daño que ha sido para el idioma español nuestro alejamiento del comercio intelectual en la época precisa en que nacieron y se constituyeron aquellos capítulos fundamentales del saber. Fueron entonces indispensables no pocos retoques de la lengua vulgar, que ciertamente no se prestaba para las nuevas preocupaciones. No sólo faltaron nombres adecuados para la designación de objetos reales o conceptos que no cabían en los moldes hechos; fue también necesario precisar relaciones que exigen flexibilidad y justeza del lenguaje utilizado en su exposición».

A modo de conclusión-objetivo escribía Cabrera: «He querido en las páginas precedentes subrayar las concepciones generales introducidas para la interpretación racional del mundo físico desde Newton, cuyas exigencias dialécticas precisan y perfeccionan el lenguaje enriqueciendo sus posibilidades de expresión. No he pretendido trazar un cuadro completo de la evolución del pensamiento físico, porque sólo quería evidenciar la conveniencia, si no la necesidad, de que en la labor de esta casa esté presente la Ciencia que desde hace más de un siglo preside el desenvolvimiento cultural».

En resumen, en esta segunda parte se ha expuesto u za colección de problemas propios del español que sólo se han destacado enunciándolos a partir de la selección de unos párrafos de Cabrera de 1936. Puede observarse que aunque antiguos no están demasiado anticuados.

\section{El panorama del Español científico: la creación y el uso de términos}

Pasemos a la tercera parte en la que pretendo exponer un poco más de cosecha propia, lo que no quiere decir original, mediante unas consideraciones de actualidad y la exhibición de algunos problemas acerca del panorama que se ofrece a la lengua española en su faceta de relación con la ciencia.

\subsection{En torno a las fuentes primarias: descubrimiento y creación}

Por lo que afecta a las fuentes primarias de terminología científica habría que decir que la primera está asociada al descubrimiento de objetos de/en la Naturaleza o a la creación de conceptos científicos, tareas propias de científicos que a continuación proceden a bautizarlos mediante su designación bien por un término, lo que sería deseable, bien por una expresión. Si hay invención hay novedad, implica evolución científica o 


\section{Francisco González de Posada}

técnica, supone innovación conceptual y/o de objeto o aparato, y, necesariamente, en consecuencia, hay que introducir una nueva voz. Por otra parte, la invención suele quedar plasmada en un artículo de una revista especializada o en un folleto de la patente correspondiente. No es fácil describir cosas nuevas con viejas palabras salvo que hubiera tal confusión que no explicara novedad, invención. De ordinario, la representación y la designación terminológica constituyen novedades.

Para la ciencia sería lamentable que no se incorporasen continuamente a ella nuevos descubrimientos y que no se crearan nuevos conceptos. Para la ingeniería sería penoso que no se inventaran nuevos ingenios. Para el lenguaje sería dramático que no lo hicieran nuevas palabras que designaran aquéllos. Complementariamente la lengua ha de ser capaz de generar en tiempo real nuevas voces que denominen las igualmente nuevas creaciones que genera la evolución científica y técnica. Una lengua moderna viva debe extender su panorama de palabras a cualquier nueva realidad. Y la lengua que no sea capaz de llevar el paso de la innovación está condenada al estancamiento, a convertirse en fósil, y este proceso de vulgarización, de antigualla, no impedirá conversaciones familiares pero será incapaz de expresar ideas complejas, concepciones novedosas, trabajos de investigación ... que exigirían en este caso la escritura y referencia a otra lengua.

La tarea de creación científica y técnica precisa de un idioma, la lengua en la que se crean los términos lingüísticos con los que se designan aquellos objetos o conceptos. Desde este punto de vista, pues, lo primario -el descubrimiento y la creación-radica en la ciencia. Y lo secundario, su formalización lingüística, el término, pertenece a la lengua. Pues bien, ambos temas, primordialmente al menos, corresponden al científico. Aquí aflora ahora parte importante de nuestro tema. Esta tarea creadora se expresa usualmente en inglés. El descubrimiento de objetos y la creación de conceptos, aunque los hicieran españoles y en España, no se publica en español, no se difunde (de ordinario) en español. En la actualidad, en España faltan tradición y contexto. Blas Cabrera y Julio Palacios fueron de otra época: otro contexto, otra tradición. Los científicos españoles actuales se han acomodado rápida y eficazmente al nuevo contexto, generando un sistema que se ha establecido como tradición en un breve período de tiempo. No estoy hablando del problema de hacer ciencia en España, que es otro problema, y ya no tan grave ni tan generalizado como en otros tiempos, sino del problema del lenguaje científico español, que es otro ciertamente.

La ciencia (nueva, la) que se hace en España se hace, aunque se considere escasa, como ciencia, pero no se hace en español, se hace en inglés. 


\section{Lenguaje y conocimiento científico}

Y los científicos españoles que hacen esta ciencia «saben" (y hasta se alardea, e incluso sólo se alardea) que «debe hacerse en inglés», porque si no es como si no se hiciera ya que no existiría -dicen-. Pero, jclaro!, el español no es, pongamos como ejemplos, ni el finlandés ni el bable; el español desempeña otro papel en la historia de la humanidad y en la cultura universal actual. No obstante, a los efectos de la ciencia parece como si no lo fuera: existe una creencia general de los científicos hispánicos que han penetrado individualmente o en pequeños grupos en el mundo de la ciencia que han asumido esta condición exacta y dócilmente. Y parece que sin retorno: entienden que es así, que debe ser así, que tiene que ser así. ¡Y hasta siempre! Y la lengua española paga las consecuencias.

\subsection{En torno al Diccionario de la Lengua Española}

La cuestión siguiente sería propiamente la de las fuentes de terminología científica española, la de los vocablos que terminarían (o quizás se iniciarían) en el Diccionario de la Lengua española. Este problema nos invita a hacer otras consideraciones.

Un diccionario se construye con "voces», «vocablos», «términos», "palabras», ... ¡Cuántos términos he utilizado ya! ¿Para un mismo -único y preciso- objeto o concepto? ¿Son sinónimos (con una pequeña diferencia) o propiamente idénticos? El Diccionario básicamente recoge las «definiciones» de los referidos términos. Y complementariamente da cabida a etimologías, notas de uso, etc.

La terminología conduce, a veces, a conflictos de muchos tipos (que suelen ser peligrosos en ocasiones por sus implicaciones jurídicas) ante las diferentes acepciones de un mismo vocablo o la existencia de diferentes vocablos (más o menos sinónimos) para la designación de un concepto. Desde una perspectiva racionalista cabría decir que cada objeto y/o cada concepto debería designarse por un término específico y ... a ser posible de tal manera que existiera un solo término para cada concepto. Así exclamaríamos: ¡Qué bien si existiera una correspondencia biunívoca entre concepto y término, entre referente y término! Pero la realidad vital de las sociedades y con ella el lenguaje -o las lenguas- han seguido el curso que han seguido. $\mathrm{Y}$ de resultas se constata que esa deseada hipotética correspondencia ... ni siquiera es unívoca. Los considerados sinónimos unas veces se manifiestan como identidades y otras auténticos inductores de confusiones. Dos términos (diferentes netamente) para un único concepto podría suponerse racionalmente absurdo, los sinónimos deberían representar más bien matices complementarios y ligeramente 
diferenciadores. Una voz designadora de diferentes conceptos (de ámbitos distantes) no crea problemas, pero si son de ámbitos próximos o del mismo sí y muchos. Suele considerarse, desde una perspectiva lingüística, riqueza de lenguaje lo que, desde otra que he sintetizado como racionalista, es también llamada a la confusión o al menos a una frecuente deficiente intelección (como se demuestra con harta frecuencia en el campo ya citado de la jurisprudencia).

La autoridad del Diccionario, en última instancia, la constituye un conjunto de personas (a veces sin relación profesional con la «ciencia» del lenguaje) que se han elegido a sí mismas, pero muchos hablantes, la mayoría y formalmente la totalidad, se ven reconocidos en y por sus decisiones. Podríamos hacer una elemental clasificación de los individuos ahora implicados que fuera simplemente orientadora: en primer lugar, los profesionales del lenguaje (filólogos, gramáticos); en segundo lugar, los usadores excepcionales del lenguaje (literatos, ensayistas); y en tercer lugar, los creadores de lenguaje (filósofos, científicos). Nuestro tema afectaría primordialmente al tercer grupo, pero ya utilizo -para el caso español- el condicional, ya que en la tesitura actual afecta, principal y directamente, a los tres grupos, como habremos detectado y cuya necesidad seguiremos constatando a medida que avancemos en nuestro discurso.

Pero el Diccionario posee una característica especial: es esencial y constitutivamente de carácter dinámico como lo es el propio lenguaje en sus debates, entre otros muchos, de enfrentamiento de voces vulgares y palabras nuevas. El lenguaje tiene, pues, naturaleza dinámica, evolutiva. Y el Diccionario, en consecuencia, análogamente.

Pero esta naturaleza dinámica se manifiesta no sólo por la mejora intrínseca siempre necesaria por ser una obra tan compleja y amplia sino, y esto es lo más llamativo sin duda, por la acción creadora de las ciencias y de las ingenierías. Son miles los términos nuevos anuales en estos ámbitos. En el caso español los neologismos y los extranjerismos constituyen probablemente lo más problemático de nuestra lengua y de nuestro Diccionario, tanto por lo incorporado como por lo ausente. Así, entre las tareas pendientes, que serían de hecho tareas relevantes permanentes por su naturaleza, podrían destacarse, por lo que respecta al lenguaje todo pero especialmente al científico, las siguientes: a) suprimir las voces arcaicas y dialectales españolas o americanas en desuso; b) ordenar las acepciones con algún criterio válido; c) introducir las nuevas voces españolas; d) «traducir» al español, inmediatamente, los términos ingleses; y e) evitar duplicaciones y circularidades.

El objetivo del Diccionario consiste en guiar al hablante nativo en la utilización de las palabras mediante unas definiciones claras y acerta- 


\section{Lenguaje y conocimiento científico}

das. Y debe tenerse una preocupación especial con la relación entre la ciencia y la técnica, por una parte, y la lengua española, por otra, en general y especialmente en las Américas. Pero sin olvidar que este problema tiene otra cara: la necesidad de mantener viva la lengua. $Y$ esto supone no olvidar que para que la lengua esté viva es básico que el universo de palabras crezca en paralelo con el de los descubrimientos y las nuevas ideas.

Es fundamental, por tanto, que todo hispanohablante disponga de un vocablo en su lengua con el que designar cualquier realidad, concepto o idea. Y que no se le condene a recurrir a barbarismos para describir realidades o conceptos innovados en otros lares o incluso en el nuestro.

Pero tampoco debe olvidarse que un diccionario pertenece también al ámbito de la política social: constituye una especie de pacto entre la instrucción y el uso. Y que de ninguna manera el pasado ha de dictar necesariamente el presente. Dinamismo abierto al futuro, apertura a la innovación, innovación en la apertura.

\subsection{En torno a las fuentes sociales}

Las relaciones del español con la ciencia, el uso del español en la ciencia se manifiesta en diferentes foros, que pueden ser principalmente los siguientes, que se comentan a modo de rápida exhibición.

1. En primer lugar deben considerarse los foros de creación de ciencia que se ubican en los ámbitos universitarios y en los específicamentę científicos. Revistas propiamente científicas en español no existen (y por tanto no se escribe en ellas, y en consecuencia no se leen, etc.). Los científicos españoles que se preciaban desde hace unas tres o cuatro décadas «tenían» que escribir en inglés y desde hace dos el sistema imperante de consideración científica y complementos económicos «los obligan» a publicar en inglés. En consecuencia, estos trabajos originales no tienen absolutamente ningún valor positivo a los efectos del lenguaje español hablado o escrito; sencillamente, no existen. La producción científica tiene lugar en lengua inglesa. Así, metafóricamente, podría decirse que cuanto más índice de impacto (mejor consideración científica pero) menor españolidad.

2. Pero, en segundo lugar, no debe olvidarse que la lengua es sobre todo uso y transmisión. Aquí se transfiere, o al menos se comparte, la responsabilidad del español científico a las ediciones de libros, revistas de divulgación, prensa, TV, radio, museos, exposiciones, etc. Hay que decir claramente que ni las bibliotecas ni las oficinas nacionales de patentes hacen 


\section{Francisco González de Posada}

la lengua, en absoluto. Y si la lengua es uso y es transmisión, la científica, por su especialización, dificultad y el estrecho margen social de su uso, lo es si se quiere más aún: ha de estar primero y sobre todo en el aula (el profesor de todos los niveles debe haber aprendido primero y transmitir en clase después los conceptos nuevos con palabras nuevas) así como en las publicaciones escritas y en los medios audiovisuales ya citados.

Quiero dejar constancia de una realidad que en la actualidad vivo diariamente. A mí me ha sorprendido extraordinariamente el hecho de que el problema objeto de análisis y de crítica en el que estamos inmersos no afecte tan drásticamente al ámbito de la Medicina. Constituye un caso particular en la Ciencia el ámbito de las ciencias médicas. Tengo una directa relación con ella, por mi participación, aunque modesta y marginal si se quiere pero intensa, en la elaboración de un Diccionario terminológico médico ${ }^{5}$. Dos notas caracterizan relativamente a la Medicina con respecto a las demás ciencias. Primera: una impresionante riqueza terminológica. Segunda: la no esclavitud tan descarada al inglés; sin duda por lo extendido e impuesto de sus raíces griegas y latinas.

3. En tercer lugar, un caso singular lo constituyen los folletos de las patentes, y a ellos, en consecuencia, debe dedicarse una atención especial. La novedad de la creación es precisamente la condición de patentabilidad. Y el folleto de una patente es, por definición, el primer contacto de la inventiva con el papel. El folleto constituye así el acto primero no propiamente de difusión pero sí de posibilitación de difusión. Las patentes son (deberían ser) un factor vigorizador del español científico y técnico, sobre todo un elemento impulsor del español tecnológico, ya que tienen su origen en la comunidad científica hispana y están redactados en nuestra lengua. Así una patente significaría un equilibrio entre el interés particular del inventor y el interés general de la comunidad. ¿Pero se difunde? De hecho mucho menos de lo que consideran los inventores de artefactos o de procesos.

Pero la Lengua es mucho más. Las revistas científicas tienen poca difusión, los artículos sólo los leen unos pocos, aunque sean los que van a seguir hablando de ello. En el caso de las patentes ocurre algo similar. A los efectos del lenguaje interesan tanto o más las fuentes secundarias ya citadas: revistas de divulgación científica, libros de texto, medios de comünicación usuales (TV, radio, prensa, etc.); en resumen las tareas que desde hace veinticinco años consideramos como de cultura científica. El contenido de las revistas científicas (vertido al español) y el de las patentes han de pasar a la Universidad, al aula, al apunte, al libro ... y al Congreso, a los medios de difusión, etc, ... ya que si no no pasa a la lengua, aunque pudiera pasar, en su caso, al Diccionario. 


\section{Lenguaje y conocimiento científico}

No obstante, no olvidemos lo científicamente primario. Patentes (en español) y artículos en revistas (inglesas) son fuentes de nuevos vocablos científicos y técnicos. Por eso hay que felicitar a los innovadores por crear ciencia e inventar técnica, pero a los primeros, además, por inventar o innovar lenguaje.

Otro elemento que no debe olvidarse es el de las patentes extranjeras que también constituyen fuentes vigorizadoras del español científico y técnico. Las patentes deben traducirse al español, como condición sine qua non, para obtener protección en España. Somos importadores netos de patentes: parece que el impacto e importancia de las traducciones se refieren al hecho de que sólo el 0,52 \% de las patentes europeas son españolas. Pero, jatención!, la Unión Europea está decidida por la no obligatoriedad de la traducción. En este caso el resultado sería análogo o quizás más grave aún para la lengua española que la costumbre de la publicación científica en inglés.

En resumen, puede concluirse, claramente y sin necesidad de matices dulcificadores, que si la situación no cambia -es decir, no la hacemos cambiar- los hispanohablantes no pueden -y no podrán- seguir el progreso, el ritmo científico y técnico de los nuevos tiempos en su idioma. Esto supone anclar la lengua española en el siglo XX, al menos en las áreas de conocimiento que implican más novedad: ciencia y técnica.

\section{A modo de sugerencias finales}

De manera específica y directa es la primera vez que me enfrento a una tarea de la naturaleza de la presente. No obstante me atrevo a sugerir, como consecuencia de las reflexiones hasta aquí explicitadas, algunas consideraciones para la acción. Detectado el grave problema que pende actualmente sobre la lengua española pueden sugerirse algunas vías para detener el proceso y corregirlo. Dos líneas principales pueden seguirse: una primera mirada se dirige a los científicos; otra, a las instituciones. Podríamos titularlas acciones recomendables.

Primera. Adquirir conciencia, todos los hispanohablantes pero especialmente los científicos, de la importancia que tiene en el mundo la lengua española, aunque ahora sólo la tengan acerca de la importancia científica de publicar en inglés. La española está llamada a ser la segunda gran lengua de comunicación del siglo XXI. En medida importante los científicos hispanos tienen la responsabilidad de que sea segunda, detrás del inglés indiscutible primera, y no sólo primera de todas las demás, que 


\section{Francisco González de Posada}

adquirirían la condición de secundarias porque sólo hubiera de hecho una relevante.

Segunda. Complementariamente, adquirir conciencia también de que la lengua española no puede permitirse renunciar a las áreas de conocimiento de la ciencia y de la técnica que constituyen sin lugar a dudas las fuentes de mayor impacto en la producción de nuevas voces. Y en el presente la lengua española está amenazada de vulgarización, de anclaje, de petrificación. Hay que superar el 'exilio científico' actual, que conduce al deterioro y al empobrecimiento de la lengua y que constituye así un perjuicio difícilmente reparable para el resto, para la lengua toda. El español de la ciencia y de la técnica debe garantizar unas nuevas fronteras del español, hoy lugar de incertidumbres y peligros; se han disparado las señales de alerta, se hace precisa la organización de la defensa y la concepción de ideas fértiles para el ataque.

Tercera. Asumir una creciente responsabilidad en el uso y en la difusión de la lengua española en los ámbitos de la ciencia y la técnica mediante la dedicación de esfuerzos a las tareas asociadas que faciliten una mayor calidad del español de la ciencia y la técnica además de garantías para el español en la ciencia y la técnica. Y esto en los diferentes marcos citados: 1) los libros científicos y técnicos en español; 2) el papel de las revistas propiamente científicas; 3) el de las revistas de divulgación; 4) el del uso en los medios, prensa, televisión, radio, museos, exposiciones, etc.; y 5) un adecuado tratamiento de las patentes. Este español de la ciencia y de la técnica constituye hoy ciertamente, por su importancia, un aparte del español, pero un aparte que repercute en el español todo, como no podía ser de otra manera, por la respectividad entre las partes que componen el todo.

Cuarta. Superar el 'desprecio' de nuestra lengua por los científicos hispanos que se manifiesta no sólo por el uso del inglés en exclusiva para sus publicaciones científicas «de prestigio», sino en la triste intelección de que así vale más el contenido ¡Qué barbaridad! Hasta ahí llega la perversión de las mentalidades. No se creen sólo que así son más leídos, que disfrutan de una mayor importancia formal y reconocimiento internacional, sino que establecen como dogma que así son mejores y sólo así son mejores. ¡Qué poca capacidad de criterio propio! ¡Qué poca confianza en lح asunción de responsabilidades para atreverse a hacer juicios personales de valor científicos!

Aquí debo traer a colación el singular ejemplo de Zubiri. Toda su obra original la escribió en español. Probablemente haya creado más conceptos y en consecuencia más términos y más acepciones que nadie. Fue libre, se hizo soberano de sí mismo, no se sometió a modas ni a modos. ¡Qué testimonio! 


\section{Lenguaje y conocimiento científico}

Quinta. Aprender sin rubor a citar a científicos (o pensadores en general) españoles por otros científicos españoles. Llama poderosamente la atención lo difícil que resulta encontrar trabajos científicos de españoles en revistas internacionales que citen a otros españoles. Y esto aunque no se leyera en el mundo. A modo de reflexión que hay que considerar en la totalidad de lo que escribo: ¿es tan importante lo que publicamos en inglés? Quizás aquí esté la cuestión. No parece que lo sen mucho ya que es prácticamente imposible leer una cita de un español en algo escrito por otro español, salvo la cita de uno mismo.

Sexta. Tampoco está de más hacer un llamamiento de acción política, mejor diríamos de acciones de gobierno, cuya perspectiva general debiera ser más abierta y de mayor responsabilidad que la de los propios científicos. Desde ahí debería decirse el «ibasta ya!» tan necesario y urgente: no a la invasión, no al sometimiento; sí al uso del español, sí al aflorar de éste en el mundo de la ciencia y de la técnica Y con acciones concretas. El Estado tiene que favorecer, financiar, fomentar la existencia de revistas científicas en español. Y premiarlas y premiar. Y con el Estado, las Autonomías, que tantas particularidades de tan diversos como estériles temas promocionan. Con carácter general conviene destacar la obligación de potenciar la expresión en español de los trabajos científicos originales.

Finalmente, a modo de grito: isin duda! isin miedos!, sí, colegas científicos. Si somos ya tan buenos que hemos ganado nuestra presencia en revistas importantes, y algunos presumen de citación en los índices solventes: ¡escribamos en español! ¡Y que nos traduzcan! ¡Y que nos lean! Y, ¡no lo olvidemos!, ... citemos a españoles en nuestros trabajos, también en los de lengua extranjera.

\section{Notas}

1 Deseo así dejar constancia de mi participación en la conmemoración de modo intelectual festivo, por una parte, y de modo crítico, por otra. Lo he citado en diferentes ocasiones en mis conferencias de ese año, pero que recuerde expresamente no he publicado, hasta ahora, una sola línea. La presente es una buena ocasión. Especialmente y con más extensión lo recordé en la de título «Blas Cabrera, académico de la Española: rigor conceptual, precisión lingüística» dictada en el III Simposio «Ciencia y Técnica en España de 1898 a 1945: Cabrera, Cajal, Torres Quevedo». Tías (Lanzarote), julio 2001. En el momento de finalizar la redacción de estas páginas ya están publicadas las Actas del I (1999) y II (2000) Simposios, con edición de F. González de Posada, F. A. González Redondo y D. 'Trujillo Jacinto del Castillo.

2 Con expresiones zubirianas, diríamos que sin aterrizar en el problema de la entificación de la realidad, por una parte, ni en el problema de la logificación de la inteligen- 


\section{Francisco González de Posada}

cia, por otra. En la bibliografía se recogen los libros más relevantes de Zubiri sobre estos temas.

3 Sobre estos temas pueden consultarse los libros de Mario Bunge (1967a, 1974a y 1974b). También el análisis recogido en la Tesis Doctoral -dirigida por mí- de González Redondo (1993).

4 Sobre la obra de Cabrera acerca de estos temas puede verse el denso estudio de González de Posada y González Redondo (1996). Un panorama general sobre el físico canario se aporta en González de Posada (1994).

5 La Real Academia Nacional de Medicina está elaborando un Diccionario terminológico en el que colaboro en las definiciones de los términos de mi especialidad.

\section{Bibliografía}

Bunge, M. (1967a): Scientific Research. New York: Springer-Verlag. [ed. esp. La investigación científica. Barcelona: Ariel. 1969. $2^{\mathrm{a}}$ ed. corregida, 1985. Trad. Miguel A. Sacristán].

Bunge, M. (1967b): Foundations of Physics. New York: Springer-Verlag.

Bunge, M. (1974a): Treatise on Basic Philosophy. Vol. I. Semantics I. Sense and Reference. Dordrecht-Boston: Reidel.

Bunge, M. (1974b): Treatise on Basic Philosophy. Vol. II. Semantics II. Interpretation and Truth. Dordrecht-Boston: Reidel.

CABRera Felipe, B. (1936): «Evolución de los conceptos físicos y lenguaje». Discurso de Ingreso. Madrid: Academia Española.

Diccionario de la Lengua Española (2001). Madrid: Real Academia Española.

GonZÁleZ DE PoSADA, F. (1992): Leonardo Torres Quevedo. Biblioteca de la Ciencia Española. Madrid: Fundación Banco Exterior.

GONZÁlEZ DE POSADA, F. (1994): Blas Cabrera: físico español, lanzaroteño ilustre. Madrid: Amigos de la Cultura Científica.

GONZÁlEZ DE POSADA, F. (2001): "Blas Cabrera: académico de la Española». Conferencia dictada en el III Simposio «Ciencia y Técnica en España de 1898 a 1945: Cabrera, Cajal, Torres Quevedo». Lanzarote, 2001. [En prensa].

GonZÁlez DE PoSAdA, F. (2001): La Física del siglo XX en la Metafísica de Zubiri. Madrid: Instituto de España.

GonzÁléz DE PosadA, F. y GonzÁlez Redondo, F. A. (1996): Blas Cabrera: Principios fundamentales de Análisis vectorial en el espacio de tres dimensiones y en el Universo de Minkowshi (1912-1913). Madrid: Amigos de la Cultura Científica.

GonZÁlez DE PosAdA, F. y GonZÁlez REDONDo, F. A. (2002): Leonardo Torres Quevedo y los dirigibles. Madrid: Intemac.

GonzÁlez Redondo, F. A. (1993): El Análisis Dimensional en la obra de Mario Bunge. Tesis Doctoral en Filosofía. Universidad Complutense de Madrid.

GONZÁlez-BuEno CATALÁN DE OCÓN, C. (2001): Patentes y lenguaje. Llamamiento contra el exilio lingüistico de la ciencia y la técnica. Madrid: Ministerio de Ciencia y Tecnología.

ORTEGA Y GASSET, J. (1930): Misión de la Universidad. [En Obras Completas, Vol. 4. Madrid: Alianza].

PAlacios MARTínez, J. (1953): «El lenguaje de la Física y su peculiar filosofía». Discurso de Ingreso. Madrid: Real Academia Española. 


\section{Lenguaje y conocimiento científico}

TORRES QUEVEDO, L. (1906): «Sobre un sistema de notaciones y símbolos destinados a facilitar la descripción de las máquinas» Revista de la Real Academia de Ciencias Exactas, Físicas y Naturales. Vol. IV, 429-442.

TORRES QUEVEDO, L. (1920): «Discurso de don Leonardo Torres Quevedo». Discurso de Ingreso. Madrid: Real Academia Española.

Vocabulario Científico y Técnico (1990). Madrid: Real Academia de Ciencias Exactas, Físicas y Naturales.

WitTGenstein, L. (1921): Tractatus logico-philosophicus [tr. e intr. J. Muñoz e I. Reguera, Alianza, Madrid, 1987].

WitTGGenstein, L. (1953): Investigaciones filosóficas. Barcelona: Crítica. [tr. A. García Suárez y U. Moulines].

ZUBIRI, X. (1962): Sobre la esencia. [5 ${ }^{a}$ ed. 1985] Madrid: Alianza.

ZUBIRI, X. (1980): Inteligencia sentiente. Madrid: Alianza.

ZUBIRI, X. (1982): Inteligencia y logos. Madrid: Alianza.

ZUBIRI, X. (1980): Inteligencia y razón. Madrid: Alianza.

ZUBIRI, X. (1989): Estructura dinámica de la realidad. Madrid: Alianza. 